\title{
Automated segmentation of leukocyte from hematological images-a study using various CNN schemes
}

\author{
Seifedine Kadry ${ }^{1} \cdot$ Venkatesan Rajinikanth $^{2}$ - David Taniar ${ }^{3}$. \\ Robertas Damaševičius ${ }^{4} \cdot$ Xiomara Patricia Blanco Valencia ${ }^{5}$ (D)
}

Accepted: 4 October 2021 / Published online: 4 November 2021

(c) The Author(s) 2021

\begin{abstract}
Medical images play a fundamental role in disease screening, and automated evaluation of these images is widely preferred in hospitals. Recently, Convolutional Neural Network (CNN) supported medical data assessment is widely adopted to inspect a set of medical imaging modalities. Extraction of the leukocyte section from a thin blood smear image is one of the essential procedures during the preliminary disease screening process. The conventional segmentation needs complex/hybrid procedures to extract the necessary section and the results achieved with conventional methods sometime tender poor results. Hence, this research aims to implement the CNN-assisted image segmentation scheme to extract the leukocyte section from the RGB scaled hematological images. The proposed work employs various CNN-based segmentation schemes, such as SegNet, U-Net, and VGG-UNet. We used the images from the Leukocyte Images for Segmentation and Classification (LISC) database. In this work, five classes of the leukocytes are considered, and each CNN segmentation scheme is separately implemented and evaluated with the ground-truth image. The experimental outcome of the proposed work confirms that the overall results accomplished with the VGG-UNet are better (Jaccard-Index $=91.5124 \%$, Dice-Coefficient $=94.4080 \%$, and Accuracy $=97.7316 \%$ ) than those of the SegNet and U-Net schemes Finally, the merit of the proposed scheme is also confirmed using other similar image datasets, such as Blood Cell Count and Detection (BCCD) database and ALL-IDB2. The attained result confirms that the proposed scheme works well on hematological images and offers better performance measure values.
\end{abstract}

Keywords Hematological images · Leukocyte segmentation · SegNet · U-Net · VGG-UNet · Performance evaluation

Xiomara Patricia Blanco Valencia xiomarapatricia.blanco@unir.net

Extended author information available on the last page of the article 


\section{Introduction}

As diseases in humans are gradually rising, an automated disease detection system is critically needed, especially when a mass screening process is essential [1-4]. Disease diagnosis with biomedical imaging is often required, in which the disease can be detected with the help of a chosen imaging modality [5, 6]. In the most chronic and infectious disease screening procedures, blood screening is a mandatory procedure, where a blood sample is collected from the patient. Detection of leukocyte (white blood cell) is particularly an essential practice during the screening of infectious diseases. This is normally performed using blood smear images (thin/thick) collected by digital microscopes [7]. Normally, hematological images collected using prescribed clinical protocol show vital information about the health condition of the patient. Further, assessment of the leukocyte count per micro-liter is also an approved practice to verify the health condition and to check the immunity level.

This work aims to develop an automated segmentation system to extract various classes of the leukocyte images available in the clinical-grade hematological test images. In the literature, a number of semi-automated/automated image processing actions have been proposed, which examined leukocyte from thin blood smear images. Our work aims to use the Convolutional Neural Network (CNN) supported segmented scheme to extract the leukocyte section with better segmentation accuracy. In the literature, a number of pre-trained and customary CNN segmentation schemes are available, which examine biomedical images recorded using varied imaging modalities [8-12]. The development of a customary CNN scheme for a chosen image is computationally complex, and hence, pre-trained CNN designs are extensively adopted by most researchers due to its availability, performance, and adaptability toward varied imaging modalities [13-17].

In this research, we adopted well-known CNN schemes, such as SegNet [9, 10], U-Net [11-13], and VGG-UNet [14-17], to extract leukocyte fragments from images with enhanced accuracy. After extracting the leukocyte fragment, a relative assessment between the extracted section and the available Ground-Truth (GT) is done to confirm the performance of the CNN scheme. The test images used in this proposed research are collected from Leukocyte Images for Segmentation and Classification (LISC) dataset [18].

This dataset consists of the five classes of leukocyte images with a dimension of $720 \times 576 \times 3$ pixels. LISC database comprised of 376 images in which 250 images are available with GT, and the remaining 126 images are without the GT. During the experimental evaluation, each image is resized into $256 \times 256 \times 3$ pixels and then the image augmentation is implemented during the training of the CNN. After the training, the performance of the CNN segmentation scheme is tested using the 250 images, which are available with the GT. The experimental investigation is separately implemented with SegNet, U-Net, and VGG-UNet. The extracted leukocyte fragment is then compared with the GT. We also calculate the Image Performance Measures (IPM), and based on these values, the performance of the CNN segmentation schemes is validated. The experimental 
outcome confirms that the CNN scheme is a promising automated segmentation technique. After pre-tuning, the VGG-UNet scheme offered a better outcome compared to SegNet and U-Net. The performance of the proposed scheme is then confirmed and validated using similar medical images existing in the literature, such as Blood Cell Count and Detection (BCCD) database and ALL-IDB2. For every image cases, the proposed scheme helps to get better segmentation accuracy and this confirms that the proposed scheme works well for hematological images. The main contribution of this research work is: (1) Employing the pretrained CNN segmentation technique to extract the abnormal section from the test image and (2) Performance evaluation of the proposed scheme on well-known benchmark images.

The remaining sections of this work are structured as follows: Sect. 2 describes the related work, Sect. 3 explains the adopted methodology, Sects. 4 and 5 present the results and conclusion of this research, respectively.

\section{Related work}

Blood screening is a commonly adopted disease prescreening procedure, and the assessment of leukocyte type and its count is also an essential practice in clinical level assessment. Due to its significance, a considerable number of leukocyte examination procedures are proposed and implemented in the literature. Table 1 presents a summary of various image processing procedures implemented to examine the hematological images.

Table 1 presents the few recently implemented leukocyte segmentation technique using traditional and DNN-based techniques. A short review of the segmentation technique employed to extract the White Blood Cell (WBC) can be found in the work of Sapna and Renuka [31]. All this work confirms that the extraction and evaluation of the WBC is a significant task during the blood level disease detection and to reduce the diagnostic burden, it is necessary to employ an automated WBC evaluation system. The recent works in the literature confirms that the CNN approaches help to achieve a superior result during the data assessment [32-35]. Hence, this research aims in implementing the $\mathrm{CNN}$ supported scheme to assess the considered database. An automated hematological image examination scheme should have the capability to detect/classify the leukocyte (WBC) with better accuracy. To achieve an automated detection, this research work employed the pre-trained CNN scheme available in the literature, and the performance of the considered CNN network is then confirmed with an experimental study using the benchmark LISC database.

\section{Methodology}

This section describes the proposed scheme, the image database used as a benchmark, the adopted CNN schemes, as well as the performance measures. 


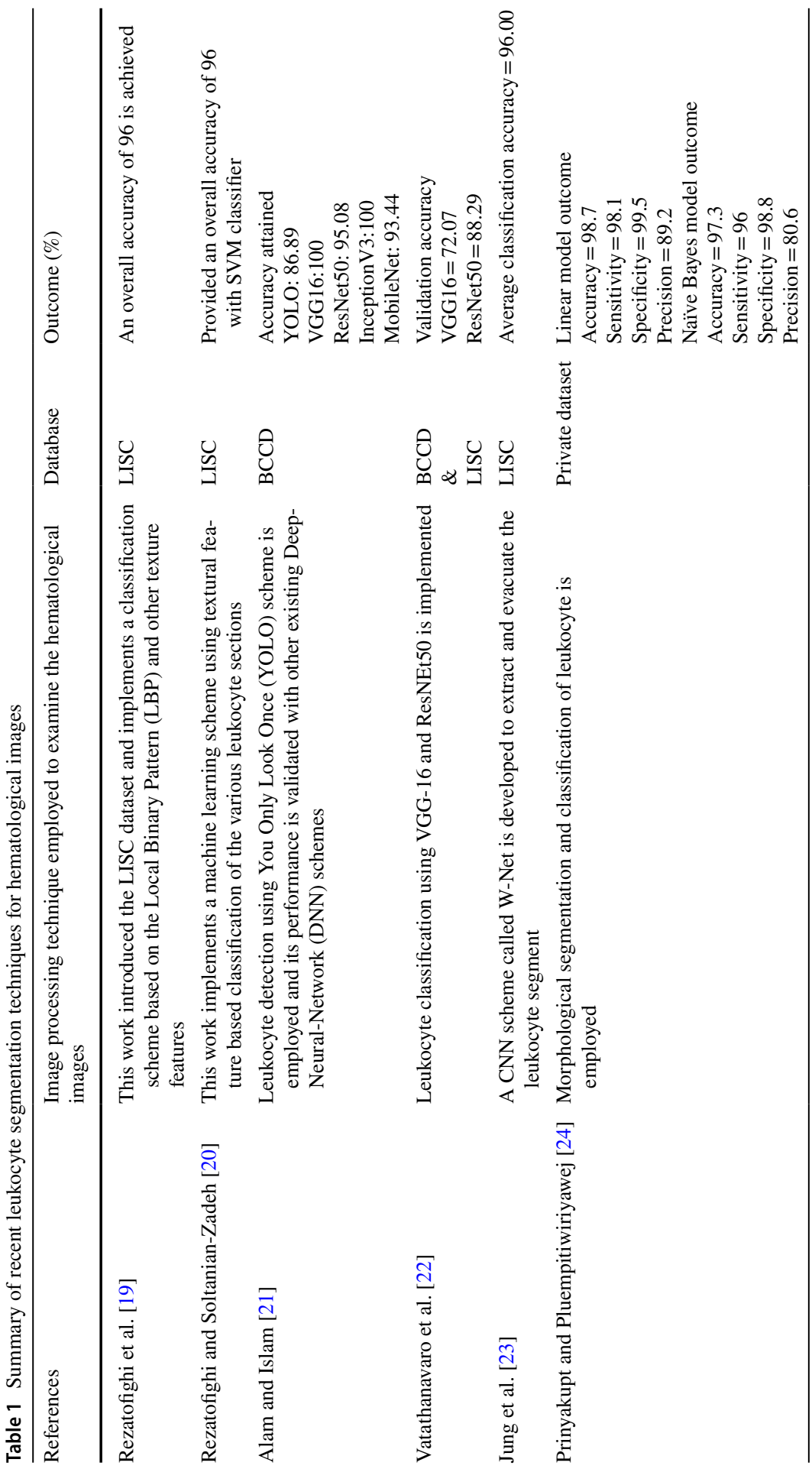




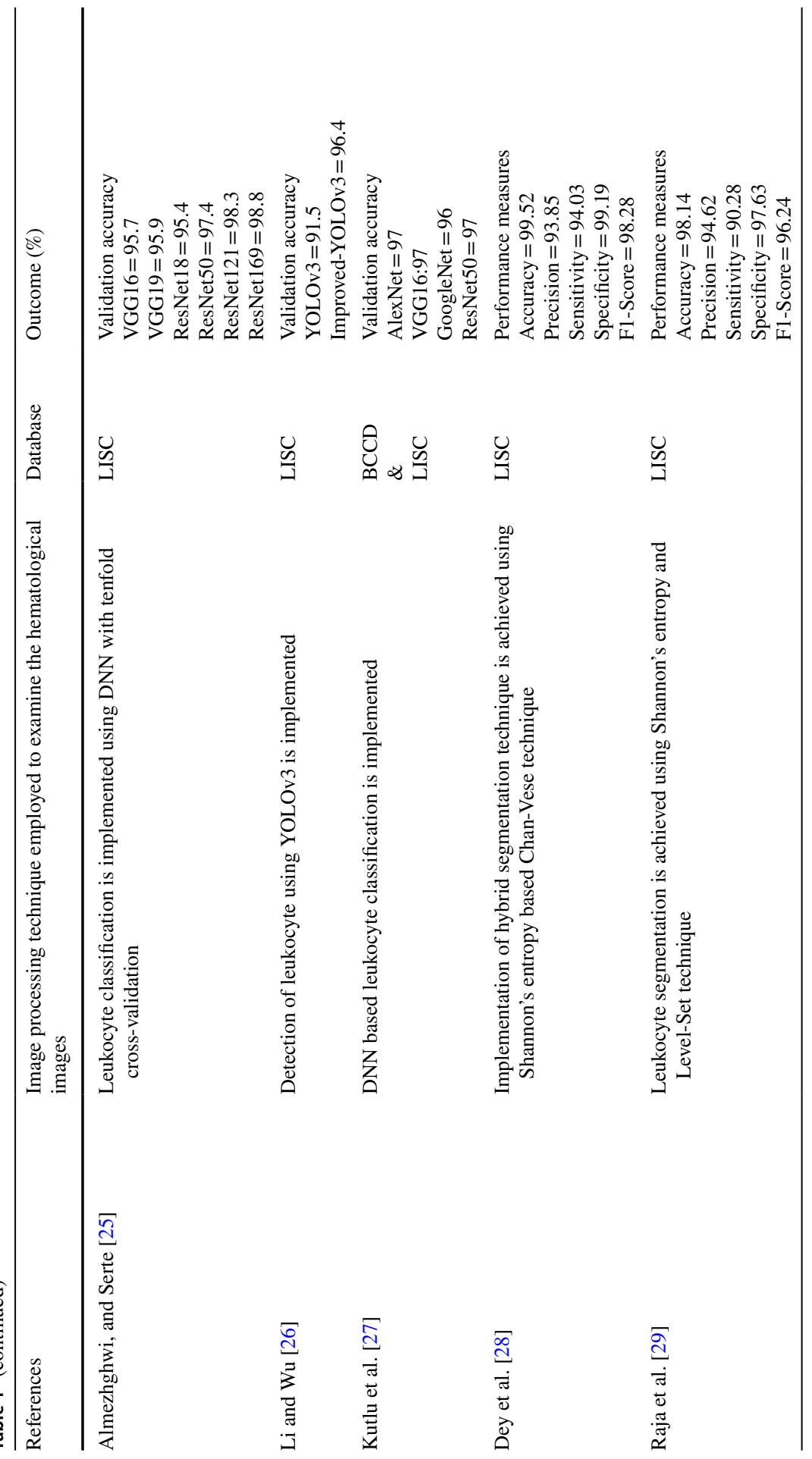




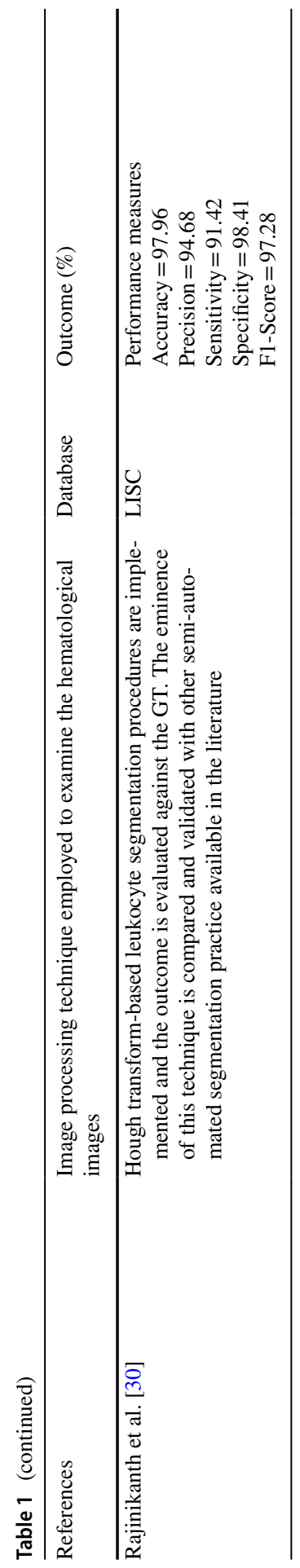




\subsection{Proposed scheme}

The structure of the proposed scheme and its stages are depicted in Fig. 1. Initially, the essential test images are gathered from the LISC database. All the RGB images of the LISC database are resized to $256 \times 256 \times 3$ pixels to reduce the computation complexity. The existing pre-trained CNN schemes, such as SegNet, U-Net, and VGG-UNet, are then used to extract the important fragment from the test images. The chosen CNN scheme is trained using the existing LISC dataset images and during this task, image augmentation, such as flip and rotate is used to increase the learning capability of the CNN. After the training process is completed, the original LISC images along with the GT (250 images) are then used to test the performance of the CNN. After image segmentation, the binary version of the segmented image is considered as the outcome and finally, this image is compared against its related GT. The results from the CNN are also compared with results from the existing literatures.

\subsection{Image database}

The development of an appropriate disease detection system is essential in the medical domain. Validating the system with the clinical-grade benchmark images is also critical. In this research, we use the LISC dataset, which is one of the clinical-grade leukocyte image datasets, developed in the year 2010 by Rezatofighi et al. [19]. This dataset consists of five categories of images, namely basophil, eosinophil, lymphocyte, monocyte, and neutrophil, as well as mixed cases. All

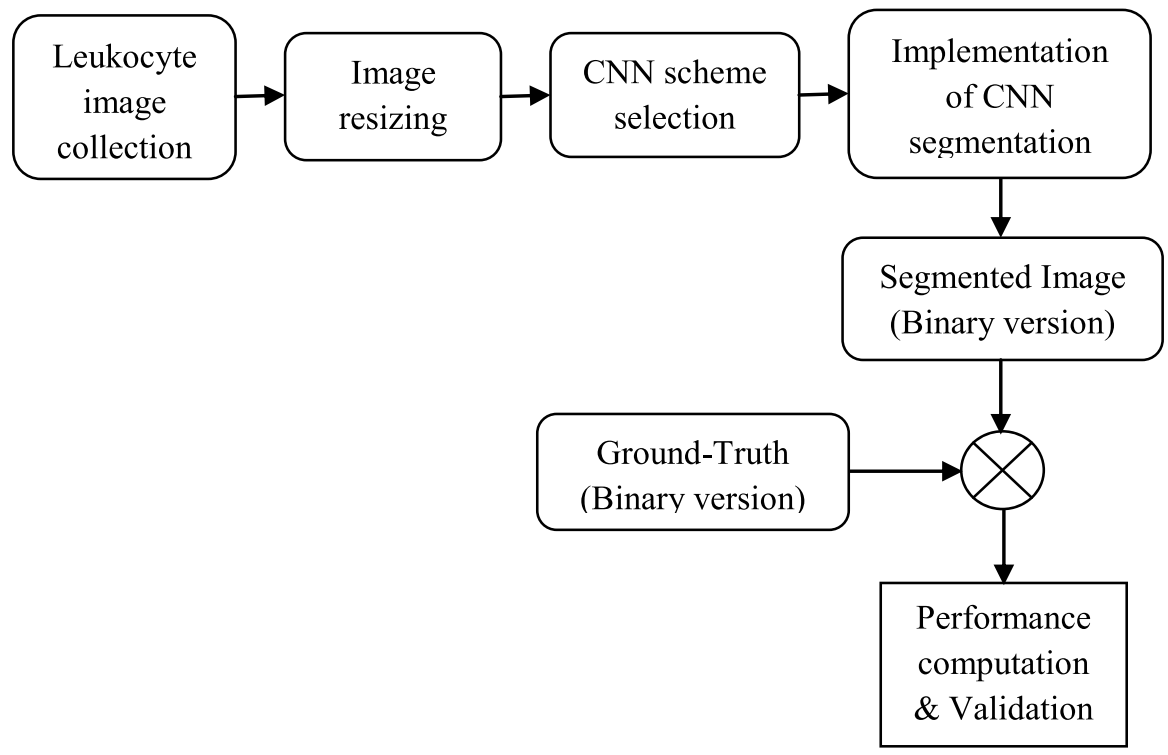

Fig. 1 Structure of the proposed CNN segmentation scheme 
these images are associated with the GT, and this dataset also has 126 images without the GT. Other related information, such as patient-related details, can be accessed from [18-20]. Figure 2 illustrates a sample test imagery of LISC with different classes.

Along with the LISC images, this research work also considers Blood Cell Count and Detection (BCCD) database [36] and ALL-IDB2 [37-39] to test the performance of the proposed scheme and the sample images from this database are depicted in Fig. 3. In this work, 250 images from each database is considered to validate the performance of the employed segmentation system.
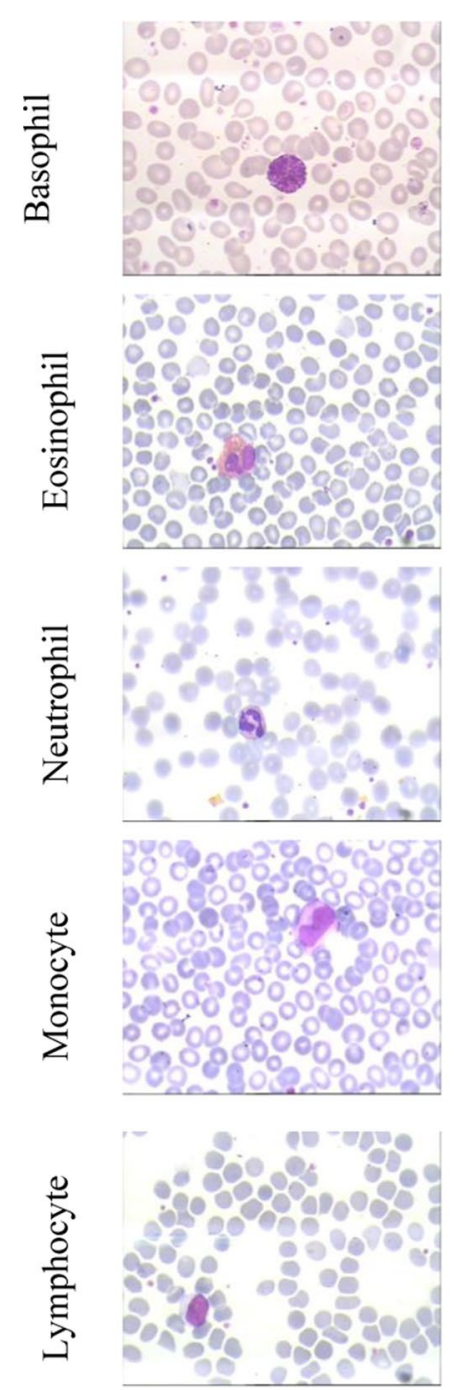
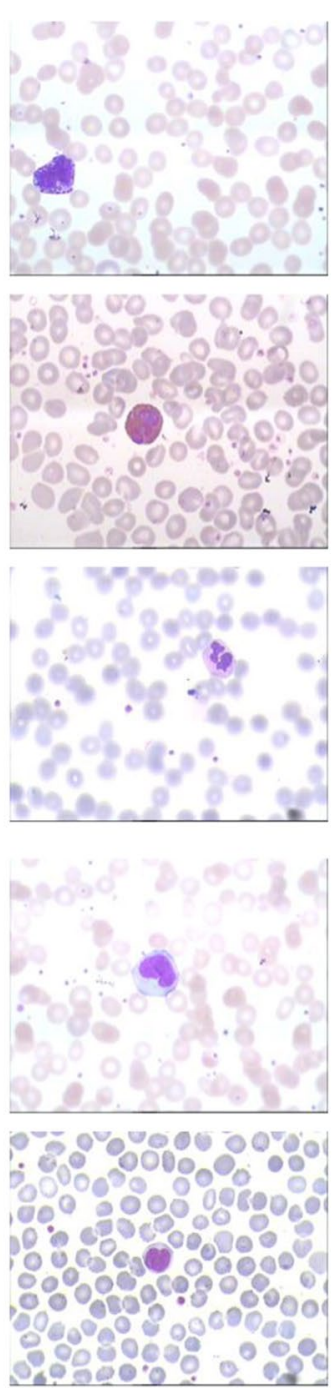
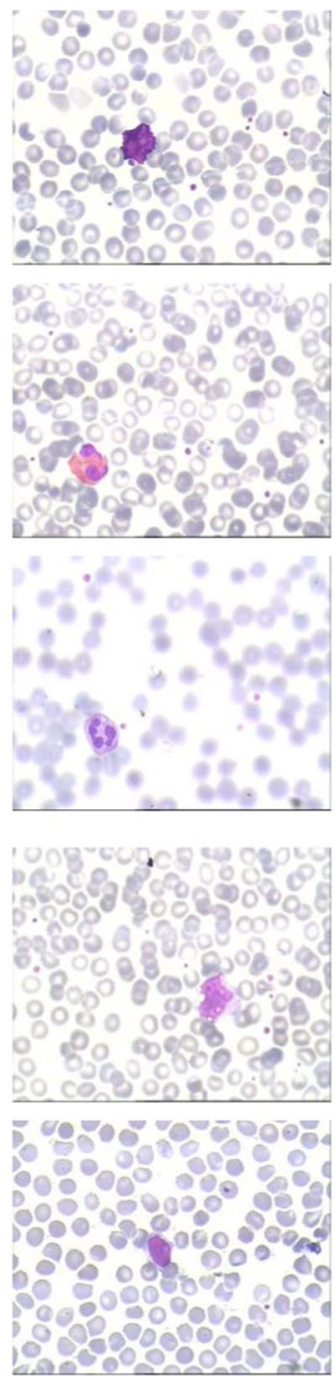

Fig. 2 Sample test images available in the LISC database 


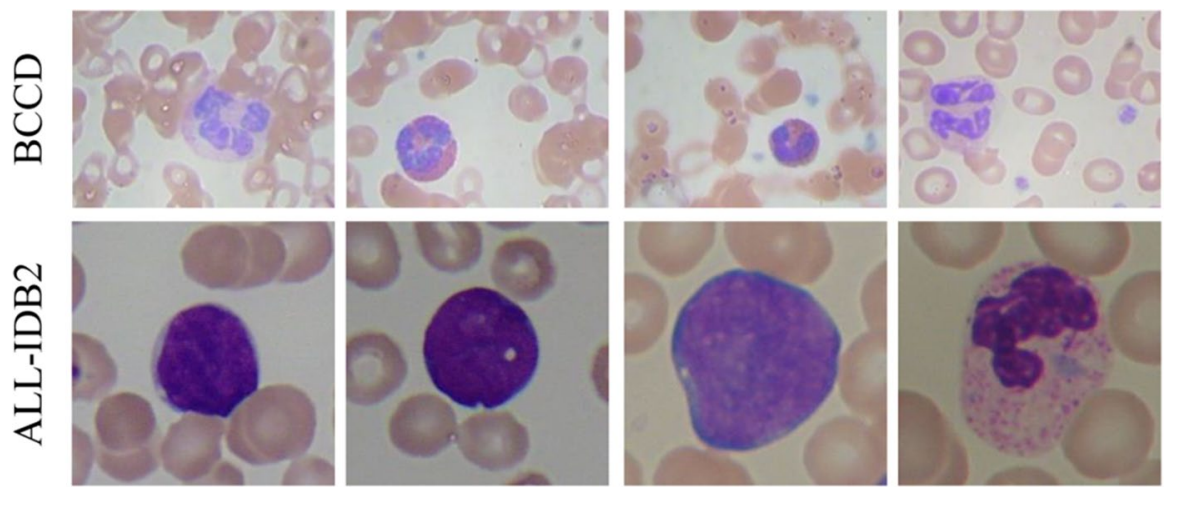

Fig. 3 Sample images of Leukocyte data considered for validation

\subsection{CNN scheme}

During the medical image diagnosis, the commonly performed image processing procedures are segmentation and classification. During the segmentation task, the essential image section (Region-Of-Interest) is extracted using a chosen technique and is then evaluated using a chosen computer algorithm to detect the disease. The development of accurate image segmentation is always essential to get better disease detection accuracy.

In the literature, a significant amount of traditional [30, 40, 41] and modern (CNN) [9, 11, 42, 43] medical segmentation techniques have been used. Implementation of the traditional segmentation techniques is time consuming, and most of the existing traditional techniques are semi-automated methods and frequently need operator assistance. Due to this reason, modern techniques are widely preferred to examine medical images of varied modalities. Recently, pre-trained CNN schemes have been extensively adopted in the image processing domain, in which the pretrained CNN schemes work well on a class of images with varied dimensions. Further, the trained $\mathrm{CNN}$ on a particular image case will produce better results compared to the traditional approaches. In this work, the CNN schemes, such as SegNet, U-Net, and VGG-UNet are considered to examine the LISC images. These CNN schemes are initially trained with the images of the LISC. During this process, the original as well as the augmented images are considered. After the training, the performance of the $\mathrm{CNN}$ is tested and validated using the leukocyte images available with the GT.

\subsubsection{SegNet}

SegNet is a well-known CNN scheme proposed in 2015. This scheme is widely used to implement the pixel-wise analysis of RGB/gray-scale images [9-11].

The SegNet is constructed by implementing a series of structured Convolutional Encoder-Decoder (CED) framework, and every framework transfers the learned 
information to the next successive section. The structure of the traditional SegNet is depicted in Fig. 4. In the implementation, we used the following parameters: image augmentation is fixed as linear, learning rate is assigned as 0.005 , decoder-encoder batch size is fixed as 4, normal weight initialization, linear dropout rate and Stochastic Gradient Descent (SGD) adaptive learning rate is considered. The last layer of this scheme is equipped with a Sigmoid activation that provides a classified binary image (which groups the pixel into two groups, such as the Leukocyte section and background). The final outcome of the SegNet is converted into a binary image in order to compare it with the binary GT.

\subsubsection{U-Net}

U-Net was proposed in 2015 as a sliding window convolutional network, dedicatedly developed to examine test images of the ISIC challenge database [12]. In recent years, due to its performance and significance, a considerable number of modified versions of U-Net schemes are available for other image databases [13-16].

The U-Net scheme used in this research is adopted from the work of El Adoui et al. [11], and the architecture is presented in Fig. 5. Here, the test image and the segmented image have the dimension of $256 \times 256 \times 3$ pixels. The initial tuning of the U-Net is the same as those for the SegNet, and are similar for the VGG-UNet scheme. The working methodology is also similar to the conventional encoderdecoder scheme. Finally, the classifier unit helps to get the outcome with two class image pixels grouped as the binary image. Other related information on the conventional U-Net can be found in [13-17].

\subsubsection{VGG-UNet}

A substantial number of CNN segmentation schemes are available in the literature, and the VGG-UNet is one of the enhanced forms of the U-Net scheme. The working

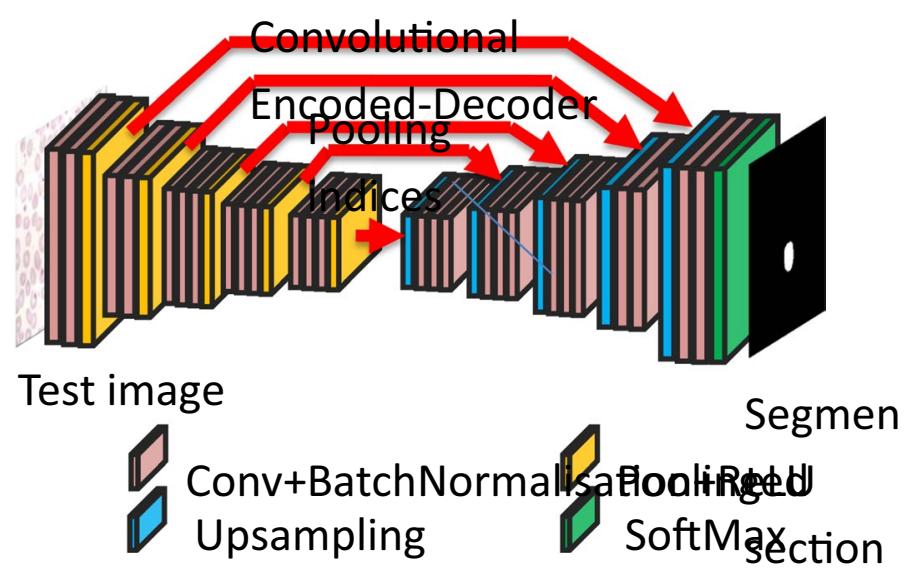

Fig. 4 Structure of the SegNet scheme 


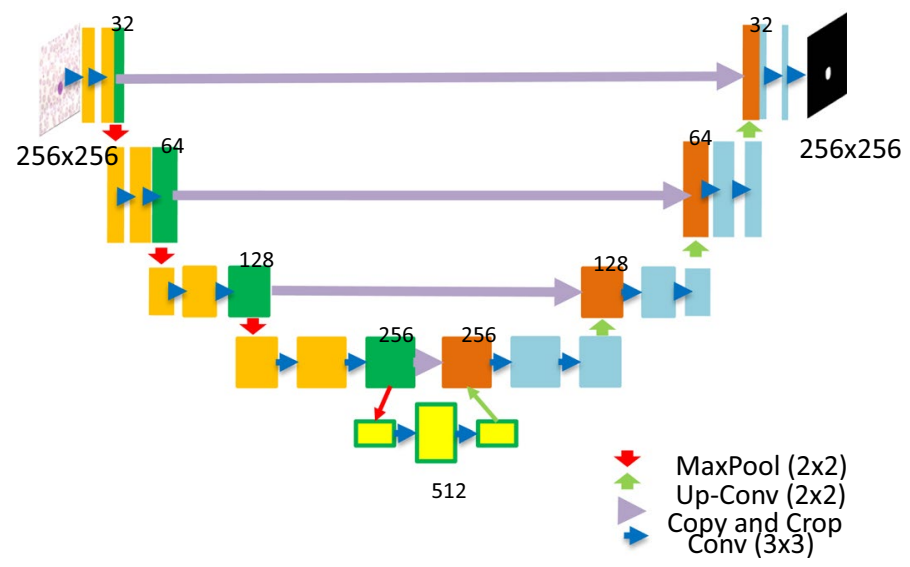

Fig. 5 Structure of the U-Net scheme

parameters and the pre-tuning of the VGG-UNet are similar to the U-Net, and in this approach, the learned features of the VGG16 scheme is considered to enhance the segmentation accuracy. During the implementation, all the examination images are resized into $224 \times 224 \times 3$ pixels. The binary image produced by this scheme is resized into $256 \times 256 x 1$ to have a fair comparison with the other CNN segmentation methods. In this work, the Convolutional part of the VGG16 will act as the encoder part and the UpConvolutional part of the U-Net act as the decoder part. Finally, the Sigmoid activation helps to get the segmented result. Other information related to the VGG-UNet can be found in the following work [13-17].

\subsection{Performance measures}

CNN segmentation performance needs to be authenticated by calculating the image performance values. After extracting the leukocyte segment from the chosen hematological image, a relative assessment with the existing GT is then performed and the essential values of the performance measures, such as Jaccard-Index (JI), DiceCoefficient (DC), Accuracy (AC), Precision (PR), Sensitivity (SE), Specificity (SP), and Negative-Predicted-Value (NPV), are calculated. Based on these values, the performance of the SegNet, U-Net, and VGG-UNet is validated. This comparison uses the binary images, in which the leukocyte region is considered as Positive $(\mathrm{P})$ pixel (binary1) and the background section is accounted as Negative (N) pixel (binary0). This comparison helps to compute the measures depicted in Eqs. (1) to (9) [45-48].

$$
\begin{gathered}
F P_{\text {rate }}=F P / N=F P /(T N+F P) \\
F N_{\text {rate }}=F N / P=F N /(T P+F N) \\
J I=F 1-\text { Score }=T P /(T P+F P+F N)
\end{gathered}
$$




$$
\begin{gathered}
D C=2 T P /(2 T P+F P+F N) \\
A C=(T P+T N) /(T P+T N+F P+F N) \\
P R=T P /(T P+F P) \\
S E=T P_{\text {rate }}=T P / P=T P /(T P+F N \\
S P=T N_{\text {rate }}=T N / N=T N /(\mathrm{TN}+\mathrm{FP}) \\
N P V=T N /(T N+F N)
\end{gathered}
$$

where $T N, T P, F N$, and $F P$ represent true-negative, true-positive, false-negative, and false-positive, respectively.

\section{Results and discussion}

After image resizing and CNN pre-tuning with the LISC dataset, the proposed segmentation is initially implemented using the test images with the GT. Figure 6 depicts the sample test image (Basophil class). Figure $6 a$, b represent the resized examination image and the related GT, respectively. Figure 6c depicts the saliency map generated by CNN during the learning process, and Fig. 6d shows the extracted binary image with the SegNet scheme. The saliency map clearly shows that CNN precisely identified the section (leukocyte) to be extracted by the final Sigmoid activation function. A similar procedure is then repeated using U-Net and VGG-UNet schemes. Their results are depicted in Fig. 6e, f, respectively. From these images, it is clearly seen that, when the $\mathrm{CNN}$ is perfectly trained with the considered image database, it identifies and segments the leukocyte section with better accuracy.

After mining the leukocyte, a number of measurements using Eqs. (1) to (9) are computed. The comparison results between Fig. 6b, d-f are shown in Tables 2 and 3. The performance measure obtained by SegNet is shown to be better than those by U-Net and the VGG-UNet. The process is then replicated to all LISC images.

The LISC dataset also consists of some complex hematological images which are challenging to many proposed computer-assisted disease detection tools, as these images are associated with more than one leukocyte section. Figure 7 shows the images with two and three leukocyte sections in a single image frame.

Figure 6 shows the image and the GT, as well as the saliency map by the SegNet scheme. The saliency map clearly confirms the correctness of the pre-training procedure implemented on the CNN segmentation using the LISC dataset. Due to its initial training, the $\mathrm{CNN}$ architecture will remember the pixel groups belong to the leukocyte section and efficiently recognizes all the pixels to be extracted. From the 


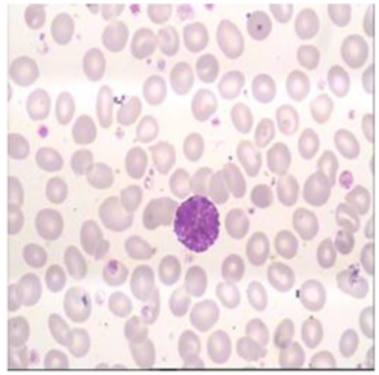

(a)

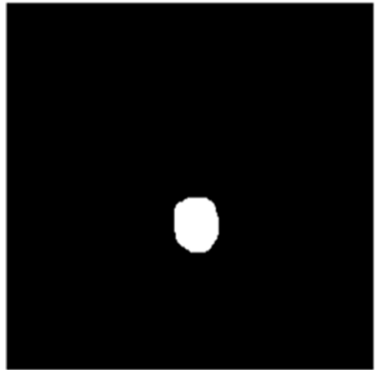

(d)

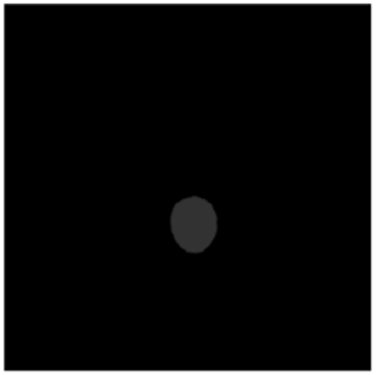

(b)

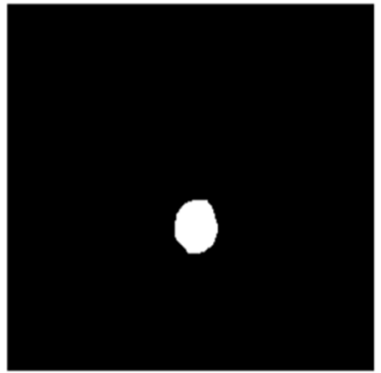

(e)

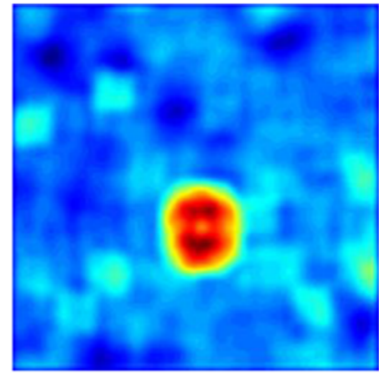

(c)

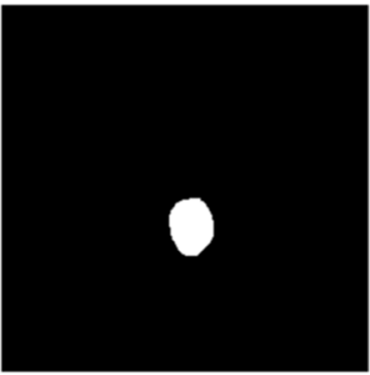

(f)

Fig. 6 Sample results using Basophil class test image Test image, b GT, c Saliency map, d-f shows extracted Leukocyte image using SegNet, U-Net, and VGG-UNet, respectively

Table 2 Performance values for the extracted leukocyte with GT

\begin{tabular}{lllllll}
\hline CNN scheme & \multicolumn{3}{l}{ Pixel level image comparison } & FP rate & FN rate \\
\cline { 2 - 5 } & TP & FP & TN & FN & & \\
\hline SegNet & 997 & 87 & 64,445 & 7 & 0.0013 & 0.0070 \\
U-Net & 884 & 200 & 64,449 & 3 & 0.0031 & 0.0034 \\
VGG-UNet & 970 & 114 & 64,441 & 11 & 0.0018 & 0.0112 \\
\hline
\end{tabular}

Table 3 Essential performance values computed using leukocyte and GT comparison

\begin{tabular}{llllllll}
\hline CNN scheme & \multicolumn{6}{l}{ Performance measures (\%) } \\
\cline { 2 - 7 } & JI & DC (F1-score) & AC & PR & SE (TP rate) & SP (TN rate) & NPV \\
\hline SegNet & 91.3841 & 95.4981 & 99.8566 & 91.9742 & 99.3028 & 99.8652 & 99.9891 \\
U-Net & 81.3247 & 89.7007 & 99.6902 & 81.5498 & 99.6618 & 99.6906 & 99.9953 \\
VGG-UNet & 88.5845 & 93.9467 & 99.8093 & 89.4834 & 98.8787 & 99.8234 & 99.9829 \\
\hline
\end{tabular}

saliency map, it is clear that the enhanced pixels belong to the leukocyte section, which will be identified and extracted by the final pixel classification layer. Approximately, similar results are obtained by both U-Net and VGG-UNet. 

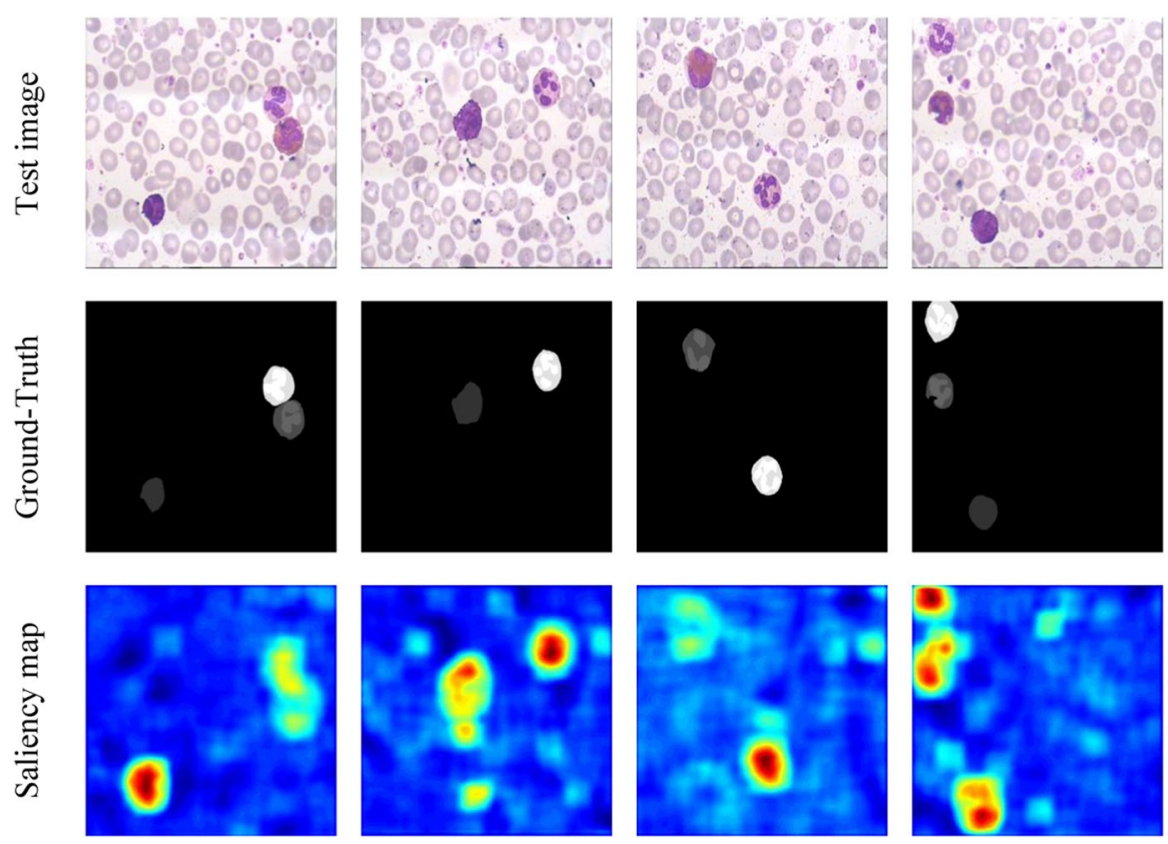

Fig. 7 Results for complex blood smear images

CNN segmentation technique is applied to the entire LISC dataset, which has the GT. The results for each leukocyte class are presented in Table 4. From this table, it is clear that SegNet, U-Net, and VGG-UNet successfully produce good image performance measures when compared with the GT, and this demonstrates that the abilities of CNN segmentation schemes.

To identify the overall performance measure, the average performance measure for each CNN scheme is separately computed. The overall measure is then compared using the Glyph-Plot [36] as shown in Fig. 8. This figure verifies that the overall performance by VGG-UNet is better compared to SegNet and U-Net. Compared to the SegNet, the traditional U-Net showed poor performance. This performance can be improved by varying the initial parameters of the pre-trained U-Net architecture.

The results obtained from this study confirm that the CNN schemes are automated schemes and work well on the leukocyte images with varied classes. Further, the overall performance measures, such as JI, DC, AC, PR, SE, SP, and NPV show promising results on each segmentation scheme.

The results of the proposed research are also evaluated against the existing semiautomated and hybrid image segmentation procedures available in the literature, and the results are presented in Figs. 9 and 10.

Figure 9 depicts the performance evaluation with Basophil image class and the results obtained by the Chan-Vese segmentation [28] technique and the CNN scheme. The Chan-Vese segmentation is used after the image thresholding process. The overall performance measure is approximately similar to the results of VGG-UNet. 
Table 4 Performance measures of individual leukocyte image class

\begin{tabular}{|c|c|c|c|c|c|c|c|c|}
\hline \multirow{2}{*}{$\begin{array}{l}\text { Image } \\
\text { class }\end{array}$} & \multirow{2}{*}{$\begin{array}{l}\text { CNN } \\
\text { scheme }\end{array}$} & \multicolumn{7}{|c|}{ Performance measures (\%) } \\
\hline & & $\mathrm{JI}$ & $\begin{array}{l}\mathrm{DC} \\
\text { (F1-Score) }\end{array}$ & $\mathrm{AC}$ & PR & $\begin{array}{l}\mathrm{SE}(\mathrm{TP} \\
\text { rate) }\end{array}$ & $\begin{array}{l}\mathrm{SP}(\mathrm{TN} \\
\text { rate) }\end{array}$ & NPV \\
\hline \multirow[t]{3}{*}{ Basophil } & SegNet & 92.0752 & 95.5517 & 97.9053 & 91.6841 & 99.2074 & 99.1653 & 99.9064 \\
\hline & U-Net & 91.0826 & 94.2281 & 97.5634 & 90.8058 & 99.2155 & 99.3106 & 99.9162 \\
\hline & VGG-UNet & 92.0452 & 95.0736 & 98.7992 & 91.2545 & 98.9551 & 99.3875 & 99.9131 \\
\hline \multirow[t]{3}{*}{ Eosinophil } & SegNet & 90.3175 & 93.0636 & 96.7395 & 91.7726 & 98.6185 & 98.3073 & 98.7716 \\
\hline & U-Net & 91.0636 & 93.7743 & 96.8116 & 91.0736 & 98.3173 & 98.1843 & 98.2284 \\
\hline & VGG-UNet & 90.8072 & 93.3327 & 97.1678 & 91.6194 & 98.2844 & 98.2005 & 98.6185 \\
\hline \multirow[t]{3}{*}{ Neutrophil } & SegNet & 91.3318 & 94.9174 & 97.7538 & 92.1073 & 98.9743 & 98.7436 & 99.2254 \\
\hline & U-Net & 90.5732 & 93.8636 & 96.9575 & 91.4974 & 98.6639 & 98.2974 & 99.3006 \\
\hline & VGG-UNet & 91.2265 & 94.1864 & 97.6937 & 92.0865 & 98.6694 & 98.8143 & 99.5495 \\
\hline \multirow[t]{3}{*}{ Monocyte } & SegNet & 91.4926 & 94.8025 & 97.4953 & 91.2286 & 98.7453 & 98.7714 & 99.3926 \\
\hline & U-Net & 92.1107 & 94.6186 & 97.2064 & 91.0375 & 98.1535 & 98.3372 & 99.6247 \\
\hline & VGG-UNet & 92.2215 & 94.8946 & 97.5927 & 91.5517 & 98.4738 & 98.8225 & 99.3279 \\
\hline \multirow{3}{*}{$\begin{array}{l}\text { Lympho- } \\
\text { cyte }\end{array}$} & SegNet & 90.7946 & 93.1667 & 97.4426 & 91.1744 & 98.9427 & 98.4227 & 98.4812 \\
\hline & U-Net & 91.1146 & 93.7268 & 96.8852 & 91.0648 & 98.3372 & 98.5924 & 98.4480 \\
\hline & VGG-UNet & 91.2615 & 94.5528 & 97.4047 & 91.3163 & 98.4074 & 98.3155 & 98.3957 \\
\hline \multirow[t]{3}{*}{ Average } & SegNet & 91.2023 & 94.3004 & 97.4673 & 91.5934 & 98.8976 & 98.6821 & 99.1554 \\
\hline & U-Net & 91.1889 & 94.0423 & 97.0848 & 91.0958 & 98.5375 & 98.5444 & 99.1036 \\
\hline & VGG-UNet & 91.5124 & 94.4080 & 97.7316 & 91.5657 & 98.5580 & 98.7081 & 99.1609 \\
\hline
\end{tabular}

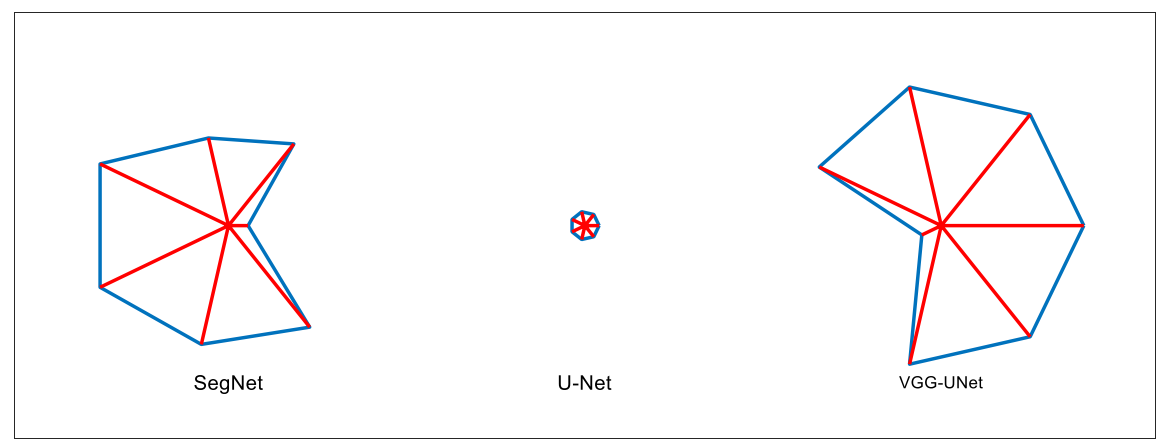

Fig. 8 Glyph-plot demonstrating the overall performance measure

Figure 10 presents a comparison result between the earlier works performed on the LISC database, such as Chan-Vese [28], Level set [29], and Hough transform [30] with proposed VGG-UNet. The earlier works discussed in the literature [28-30] are hybrid image processing methods, in which the preprocessing is performed using a heuristic algorithm-assisted thresholding procedure, and mining is employed with the chosen segmentation technique. The overall accuracy by the VGG-UNet is 


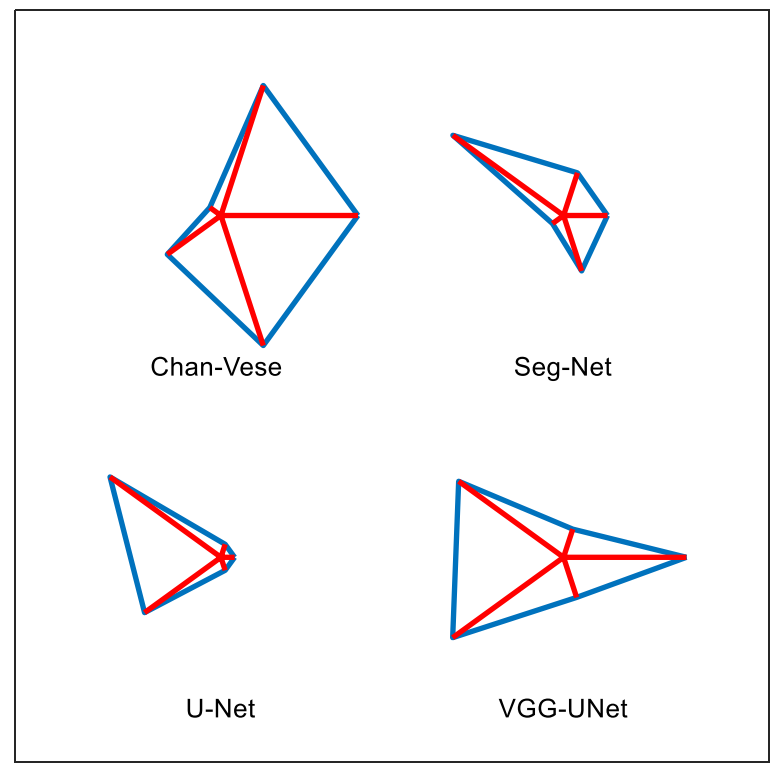

Fig. 9 Glyph-Plot of the overall performance measures obtained with Basophil image class

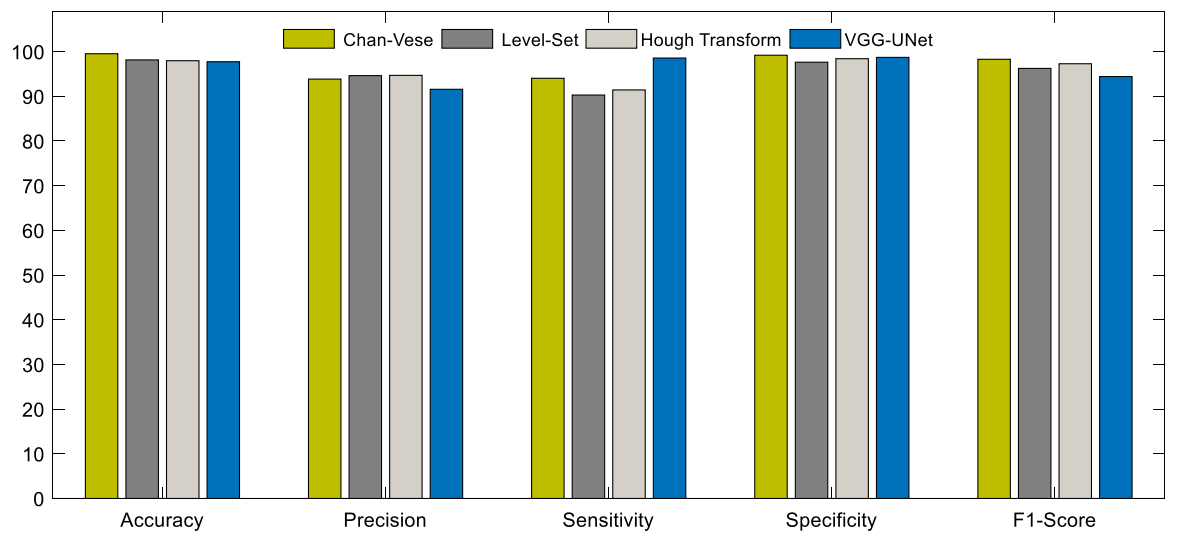

Fig. 10 Performance evaluation between VGG-UNet with existing semi-automated and hybrid image processing schemes

quite similar to that of the existing methods, and sensitivity and specificity are better compared to the existing traditional procedures.

Figure 11 presents the sample results achieved with VGG-UNet, and this result confirms that the outcome in both BCCD and ALL-IDB2 database is good. This result is compared with the segmentation result of VGG-SegNet, and the outcome is presented in Fig. 12. From this figure, it can be noted that, the VGG-UNet based segmentation helps to get a better average values of JI, DC, and AC compared to 

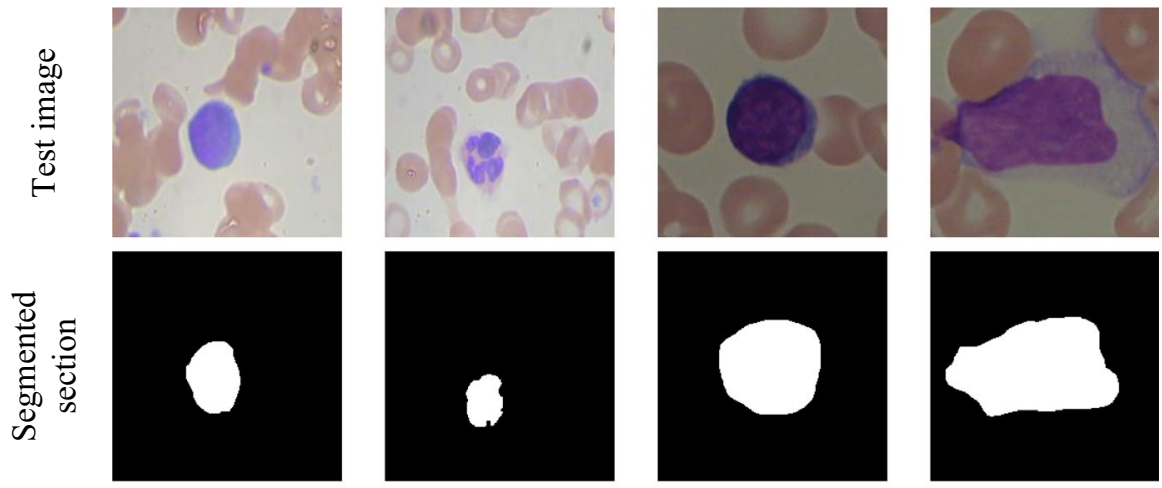

Fig. 11 Results achieved with BCCD and ALL-IDB2

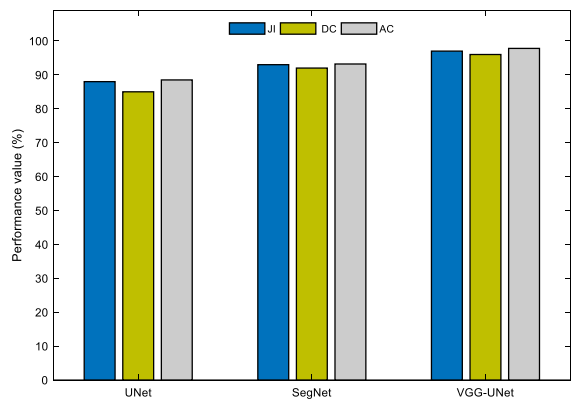

(a) Results for BCCD

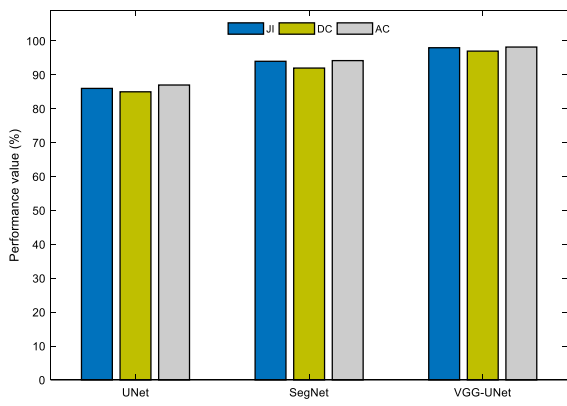

(b) Results for ALL-IDB2

Fig. 12 Comparison of average performance achieved for 250 images

UNet, SegNet, and VGG-SegNet. Figure 12a presents the BCCD database outcome, and Fig. 12b shows the ALL-IDB2 image result. These results confirm that the VGG-UNet helps to get a comparatively better outcome on both the datasets.

The future scope of our research work may be concentrated toward improving the performance of the CNN schemes by adjusting the initial parameters, such as adjusting the image augmentation process, improving the learning, modifying the decoder-encoder batch, adjusting the weight initialization process, modifying the dropout rate, and modifying the activation layer.

This work employed encoder-decoder (VGG-UNet) scheme to achieve accurate segmentation of the leukocyte region in RGB-scaled image. The outcome of the encoder section will be the learned features (Deep-Features), and this feature can be considered to classify the images using a binary or multiclass classifiers. The future scope of this research includes: (1) Implementation of VGG supported automated image classification, (2) Development of VGG-SegNet, and (3) Examination of clinical-grade images. 


\section{Conclusion}

Due to its medical importance, a significant amount of image assessment schemes is planned and implemented by the researches for the medical images with varied modalities. This research presented and automated leukocyte extraction system from hematological images using the benchmark LISC dataset. This work used the pretrained CNN segmentation procedures, such as SegNet, U-Net, and VGG-UNet to extort the leukocyte section with better accuracy. The proposed segmentation procedure is implemented on 250 images associated with the GT and before implementing the segmentation process, every test image is resized into $256 \times 256 \times 3$ pixels, in order to reduce the computation burden. The results are then compared to identify which CNN scheme produced the best outcome for the LISC dataset. Our experimental results show that the VGG-UNet produced better results than SegNet and U-Net. The outcome of the VGG-UNet is also authenticated against the other hybrid segmentation procedures existing in the literature. Further, the eminence of proposed scheme is tested and validated on BCCD and ALL-IDB2 and these results also verify that this technique helps to segment the leukocyte image perfectly. This research work demonstrated that CNN is useful and is significant in the clinical domain. In future, we will examine the clinical grade of hematological images. Furthermore, the proposed VGG-UNet approach can also be considered to classify the considered image database with a binary and multiclass classifier to support the automated leukocyte class recognition.

Open Access This article is licensed under a Creative Commons Attribution 4.0 International License, which permits use, sharing, adaptation, distribution and reproduction in any medium or format, as long as you give appropriate credit to the original author(s) and the source, provide a link to the Creative Commons licence, and indicate if changes were made. The images or other third party material in this article are included in the article's Creative Commons licence, unless indicated otherwise in a credit line to the material. If material is not included in the article's Creative Commons licence and your intended use is not permitted by statutory regulation or exceeds the permitted use, you will need to obtain permission directly from the copyright holder. To view a copy of this licence, visit http://creativecommons.org/licen ses/by/4.0/.

\section{References}

1. Fernandes SL, Rajinikanth V, Kadry S (2019) A hybrid framework to evaluate breast abnormality using infrared thermal images. IEEE Con Electron Mag 8(5):31-36. https://doi.org/10.1109/MCE. 2019.2923926

2. Fernandes SL, Tanik UJ, Rajinikanth V, Karthik KA (2019) A reliable framework for accurate brain image examination and treatment planning based on early diagnosis support for clinicians. Neural Comput Appl. https://doi.org/10.1007/s00521-019-04369-5

3. Ahilan A, Chandra Babu G, Senthil Murugan N, Parthasarathy MG, Raja C, Kadry S, Kumar SN, Agees Kumar C, Jarin T, Krishnamoorthy S, Malarvizhi Kumar P (2019) Segmentation by fractional order Darwinian particle swarm optimization based multilevel thresholding and improved lossless prediction based compression algorithm for medical images. IEEE Access 7:89570-89580. https://doi.org/10.1109/ACCESS.2019.2891632 
4. Hussain UN, Khan MA, LaliI U, Javed K, Ashraf I, Tariq J et al (2020) A unified design of ACO and skewness based brain tumor segmentation and classification from MRI scans. Control Eng Appl Inform 22(2):43-55

5. Bakiya A, Kamalanand K, Rajinikanth V, Nayak RS, Kadry S (2020) Deep neural network assisted diagnosis of time-frequency transformed electromyograms. Multimedia Tool Appl 79(1516):11051-11067. https://doi.org/10.1007/s11042-018-6561-9

6. Kadry S (2020) An efficient apriori algorithm for frequent pattern mining using mapreduce in healthcare data. BullElectrEngInform 10(1):390-403

7. Sharif M, Amin J, Siddiqa A, Khan HU, Arshad Malik MSA, Anjum MA, Kadry S (2020) Recognition of different types of leukocytes using YOLOv2 and optimized bag-of-features. IEEE Access 8:167448-167459. https://doi.org/10.1109/ACCESS.2020.3021660

8. Rajinikanth V, Joseph Raj AN, Thanaraj KP, Naik GR (2020) A customized VGG19 network with concatenation of deep and handcrafted features for brain tumor detection. Appl Sci 10(10):3429. https://doi.org/10.3390/app10103429

9. Badrinarayanan V, Handa A, Cipolla R (2015) Segnet: A deep convolutional encoder-decoder architecture for robust semantic pixel-wise labelling. arXiv preprint arXiv:1505.07293

10. Badrinarayanan V, Kendall A, Cipolla R (2017) Segnet: a deep convolutional encoder-decoder architecture for image segmentation. IEEE Trans Pattern Anal Mach Intell 39(12):2481-2495. https://doi.org/ 10.1109/TPAMI.2016.2644615

11. El Adoui M, Mahmoudi SA, Larhmam MA, Benjelloun M (2019) MRI breast tumor segmentation using different encoder and decoder CNN architectures. Computers 8(3):52. https://doi.org/10.3390/ computers 8030052

12. Ronneberger O, Fischer P, Brox T (2015) U-net: convolutional networks for biomedical image segmentation. Lecture Notes in Computer Science International Conference on Medical Image Computing and Computer-Assisted Intervention, pp 234-41. https://doi.org/10.1007/978-3-319-24574-4_28

13. Fawakherji M, Youssef A, Bloisi D, Pretto A, Nardi D (2019) Crop and weeds classification for precision agriculture using context-independent pixel-wise segmentation. In: Third IEEE International Conference on Robotic Computing (IRC), IEEE Publications 2019, pp 146-152. https://doi.org/10.1109/ IRC.2019.00029

14. Pravitasari AA, Iriawan N, Almuhayar M, Azmi T, Irhamah I, Fithriasari K, Purnami SW, Ferriastuti W (2020) UNet-VGG16 with transfer learning for MRI-based brain tumor segmentation. Telkomnika 18(3):1310-1318. https://doi.org/10.12928/telkomnika.v18i3.14753

15. Iglovikov V, Shvets A (2018) Ternausnet: U-net with VGG11 encoder pre-trained on imagenet for image segmentation. arXiv preprint arXiv:1801.05746

16. Frid-Adar M, Ben-Cohen A, Amer R, Greenspan H (2018) Improving the segmentation of anatomical structures in chest radiographs using U-Net with an imagenet pre-trained encoder. Lecture Notes in Computer Science, pp159-168. https://doi.org/10.1007/978-3-030-00946-5_17

17. Iglovikov V, Seferbekov SS, Buslaev A, Shvets A (2018) TernausNetV2: fully convolutional network for instance segmentation. CVPR Workshops p 233. https://doi.org/10.1109/CVPRW.2018.00042

18. http://users.cecs.anu.edu.au/ hrezatofighi/Data/Leukocyte\%20Data.htm

19. Rezatofighi SH, Khaksari K, Soltanian-Zadeh H (2010) Automatic recognition of five types of white blood cells in peripheral blood. International Conference Image Analysis and Recognition, pp 161-172. https://doi.org/10.1007/978-3-642-13775-4_17

20. Rezatofighi SH, Soltanian-Zadeh H (2011) Automatic recognition of five types of white blood cells in peripheral blood. ComputMed ImagingGraph 35(4):333-343. https://doi.org/10.1016/j.compmedimag. 2011.01.003

21. Alam MM, Islam MT (2019) Machine learning approach of automatic identification and counting of blood cells. Healthc Technol Lett 6(4):103-108. https://doi.org/10.1049/htl.2018.5098

22. Vatathanavaro S, Tungjitnob S, Pasupa K White blood cell classification: a comparison between VGG16 and ResNet-50 models

23. Jung C, Abuhamad M, Alikhanov J, Mohaisen A, Han K, Nyang D (2019) W-net: a CNN-based architecture for white blood cells image classification. arXiv preprint arXiv:1910.01091

24. Prinyakupt J, Pluempitiwiriyawej C (2015) Segmentation of white blood cells and comparison of cell morphology by linear and naïve Bayes classifiers. Biomed Eng OnLine 14(1):63. https://doi.org/10. 1186/s12938-015-0037-1

25. Almezhghwi K, Serte S (2020) Improved classification of white blood cells with the generative adversarial network and deep convolutional neural network. Comput Intell Neurosci 2020:6490479. https:// doi.org/10.1155/2020/6490479 
26. Li J, Wu J (2020) Leukocyte detection in blood smear image based on improved YOLOv3. In: Proceedings of the 2020 the 10th International Workshop on Computer Science and Engineering (WCSE 2020), pp 144-149. https://doi.org/10.18178/wcse.2020.06.024

27. Kutlu H, Avci E, Özyurt F (2020) White blood cells detection and classification based on regional convolutional neural networks. Med Hypotheses 135:109472. https://doi.org/10.1016/j.mehy.2019.109472

28. Dey N, Shi F, Rajinikanth V (2019) Leukocyte nuclei segmentation using entropy function and Chan-Vese approach. Inf Technol Intell Transp Syst 314: 255-264.. https://doi.org/10.3233/ 978-1-61499-939-3-255

29. Raja NSM, Arunmozhi S, Lin H, Dey N, Rajinikanth V (2019) A study on segmentation of leukocyte image with Shannon's entropy. Adv Med Technol Clin Pract pp 1-27. https://doi.org/10.4018/978-15225-6316-7.ch001

30. Rajinikanth V, Dey N, Kavallieratou E, Lin H (2020) Firefly algorithm-based Kapur's thresholding and Hough transform to extract leukocyte section from hematological images. Springer Tracts in NatureInspired Computing, pp 221-235. https://doi.org/10.1007/978-981-15-0306-1_10

31. Sapna S, Renuka A (2017) Techniques for segmentation and classification of leukocytes in blood smear images-a review. In: IEEE International Conference on Computational Intelligence and Computing Research (ICCIC). Vol. 2017. IEEE Publications; 2017, December. pp 1-5. https://doi.org/10.1109/ ICCIC.2017.8524465

32. Song H, Han X-Y, Montenegro-Marin CE, Krishnamoorthy S (2021) Secure prediction and assessment of sports injuries using deep learning based convolutional neural network. J Ambient Intell Hum Comput 12(3):3399-3410. https://doi.org/10.1007/s12652-020-02560-4

33. Bobadilla J, Ortega F, Gutiérrez A, Alonso S (2020) Classification-based deep neural network architecture for collaborative filtering recommender systems. Int J Interact Multimedia Artif Intell 6(1):68-77. https://doi.org/10.9781/ijimai.2020.02.006

34. Lin JCW, Shao Y, Djenouri Y, Yun U (2021) ASRNN: a recurrent neural network with an attention model for sequence labeling. Knowl Based Syst 212:106548. https://doi.org/10.1016/j.knosys.2020. 106548

35. Maheshan MS, Harish BS, Nagadarshan N (2020) A convolution neural network engine for sclerare cognition. Int J Interact Multimedia Artif Intell 6(1):78-83. https://doi.org/10.9781/ijimai.2019.03.006

36. https://www.kaggle.com/surajiiitm/bccd-dataset

37. https://www.kaggle.com/nikhilsharma00/leukemia-dataset

38. Donida Labati R, Piuri V, Scotti F (2011) ALL-IDB: the acute lymphoblastic leukemia image database for image processing. In: Proceedings of the 2011 IEEE International Conference on Image Processing (ICIP 2011), Brussels, Belgium, pp 2045-2048. https://doi.org/10.1109/ICIP.2011.6115881

39. Scotti F (2006) Robust segmentation and measurements techniques of white cells in blood microscope images. In: Proceedings of the 2006 IEEE Instrumentation and Measurement Technology Conference (IMTC 2006), Sorrento, Italy, pp 43-48. https://doi.org/10.1109/IMTC.2006.328170

40. Kasihmuddin MSBM, Mansor MAB, Abdulhabib Alzaeemi S, Sathasivam S (2021) Satisfiability logic analysis via radial basis function neural network with Artificial Bee Colony Algorithm. Int J Interact Multimedia Artif Intell 6(6):164-173. https://doi.org/10.9781/ijimai.2020.06.002

41. Devi SS, Singh NH, Laskar RH (2020) Fuzzy C-means clustering with histogram based cluster selection for skin lesion segmentation using non-dermoscopic images. Int J Interact Multimedia Artif Intell 6(1):26-31. https://doi.org/10.9781/ijimai.2020.01.001

42. Khari M, Garg AK, Gonzalez-Crespo RG, Verdú E (2019) Gesture recognition of RGB and RGB-D staticimages using convolutional neuralnetworks. Int J Interact Multimedia Artif Intell 5(7):22-27. https://doi.org/10.9781/ijimai.2019.09.002

43. Robinson YH, Vimal S, Khari M, Hernández FCL, Crespo RG (2020) Tree-based convolutional neural networks for object classification in segmented satellite images. Int J High Perform Comput Appl. https://doi.org/10.1177/1094342020945026

44. Pugalenthi R, Rajakumar MP, Ramya J, Rajinikanth V (2019) Evaluation and classification of the brain tumor MRI using machine learning technique. Control Eng Appl Inform 21(4):12-21

45. Dey N, Rajinikanth V, Shi F, Tavares JMRS, Moraru L, Arvind Karthik KA, Lin H, Kamalanand K, Emmanuel C (2019) Social-Group-Optimization based tumor evaluation tool for clinical brain MRI of Flair/diffusion-weighted modality. Biocybern Biomed Eng 39(3):843-856. https://doi.org/10.1016/j. bbe.2019.07.005

46. Wang Y, Chen Y, Yang N, Zheng L, Dey N, Ashour AS, Rajinikanth V, Tavares JMRS, Shi F (2019) Classification of mice hepatic granuloma microscopic images based on a deep convolutional neural network. Appl Soft Comput 74:40-50. https://doi.org/10.1016/j.asoc.2018.10.006 
47. Mani MS, Manisha S, Thanaraj KP, Rajinikanth V (2017) Automated segmentation of Giemsa stained microscopic images based on entropy value. In: International Conference on Intelligent Computing, Instrumentation and Control Technologies (ICICICT). IEEE Publications; 2017, pp. 1124-1128. https://doi.org/10.1109/ICICICT1.2017.8342727

48. Wickham H, Hofmann H, Wickham C, Cook D (2012) Glyph-maps for visually exploring temporal patterns in climate data and models. Environmetrics 23(5):382-393. https://doi.org/10.1002/env.2152

Publisher's Note Springer Nature remains neutral with regard to jurisdictional claims in published maps and institutional affiliations.

\section{Authors and Affiliations}

\section{Seifedine Kadry ${ }^{1}$. Venkatesan Rajinikanth ${ }^{2}$ - David Taniar ${ }^{3}$. Robertas Damaševičius ${ }^{4}$ Xiomara Patricia Blanco Valencia ${ }^{5}$}

Seifedine Kadry

Seifedine.kadry@noroff.no

Venkatesan Rajinikanth

v.rajinikanth@ieee.org

David Taniar

David.Taniar@monash.edu

Robertas Damaševičius

robertas.damasevicius@ktu.lt

1 Faculty of Applied Computing and Technology, Noroff University College, Kristiansand, Norway

2 Department of Electronics and Instrumentation Engineering, St. Joseph's College of Engineering, OMR, Tamil Nadu, Chennai 600119, India

3 Faculty of Information Technology, Monash University, Clayton Campus, Melbourne, VIC 3800, Australia

4 Faculty of Applied Mathematics, Silesian University of Technology, 44-100, Gliwice, Poland

5 Computer Science and Technology Area, Universidad Internacional de La Rioja (UNIR), Logroño, Spain 